\title{
An Individual Patient Data Meta-Analysis of the Long-Term Outcome of Randomised Studies Comparing Intravesical Mitomycin C versus Bacillus Calmette-Guérin for Non-Muscle-Invasive Bladder Cancer
}

\author{
Per-Uno Malmström ${ }^{a, *}$, Richard J. Sylvester ${ }^{b}$, David E. Crawford ${ }^{c}$, Martin Friedrich $^{d}$, \\ Susanne Krege ${ }^{e}$, Erkki Rintala $^{f}$, Eduardo Solsona $^{g}$, Savino M. Di Stasi $^{h}$, J. Alfred Witjes $^{i}$ \\ a Uppsala University Hospital, Department of Urology, Uppsala, Sweden \\ ${ }^{\mathrm{b}}$ European Organisation for Research and Treatment of Cancer Headquarters, Brussels, Belgium \\ ${ }^{\mathrm{c}}$ University of Colorado, Department of Urology, Denver, CO, USA \\ ${ }^{\mathrm{d}}$ Helios Klinikum Krefeld, Department of Urology, Krefeld, Germany \\ ${ }^{\mathrm{e}}$ KH Maria Hilf, Department of Urology, Krefeld, Germany \\ ${ }^{\mathrm{f}}$ Helsinki University Hospital, Department of Urology, Helsinki, Finland \\ ${ }^{\mathrm{g}}$ Servicio de Urología, Instituto Valenciana de Oncologia, Valencia, Spain \\ h "Tor Vergata" University, Department of Urology, Rome, Italy \\ ${ }^{\mathrm{i}}$ Radboud University, Department of Urology, Nijmegen, Netherlands
}

Article info

Article history:

Accepted April 16, 2009

Published online ahead of print on April 24, 2009

Keywords:

Bladder neoplasms

Meta-analysis

Instillation therapy

Mitomycin-C

Bacillus Calmette-Guérin

\begin{abstract}
Background: Patients with non-muscle-invasive bladder cancer with an intermediate or high risk need adjuvant intravesical therapy after surgery. Based largely on meta-analyses of previously published results, guidelines recommend using either bacillus Calmette-Guérin (BCG) or mitomycin $C$ (MMC) in these patients. Individual patient data (IPD) meta-analyses, however, are the gold standard.

Objective: To compare the efficacy of BCG and MMC based on an IPD meta-analysis of randomised trials.

Design, setting, and participants: Trials were searched through Medline and review articles. The relevant trial investigators were contacted to provide IPD.

Measurements: The drugs were compared with respect to time to recurrence, progression, and overall and cancer-specific death.

Results and limitations: Nine trials that included 2820 patients were identified, and IPD were obtained from all of them. Patient characteristics were $71 \%$ primary, $54 \%$ $\mathrm{Ta}, 43 \% \mathrm{~T} 1,25 \% \mathrm{G} 1,58 \% \mathrm{G} 2$, and $16 \% \mathrm{G} 3$, and $7 \%$ had prior intravesical chemotherapy. Based on a median follow-up of $4.4 \mathrm{yr}$, $43 \%$ recurred. Overall, there was no difference in the time to first recurrence ( $p=0.09$ ) between BCG and MMC. In the trials with BCG maintenance, a $32 \%$ reduction in risk of recurrence on BCG compared to MMC was found ( $p<0.0001$ ), while there was a $28 \%$ risk increase $(p=0.006)$ for BCG in the trials without maintenance. BCG with maintenance was more effective than MMC in both patients previously treated and those not previously treated with

* Corresponding author. University Hospital, Urology, Sjukhusvägen 1, Uppsala, 75185, Sweden. E-mail address: per-uno.malmstrom@surgsci.uu.se (P.-U. Malmström).
\end{abstract}


chemotherapy. In the subset of 1880 patients for whom data on progression, survival, and cause of death were available, $12 \%$ progressed and $24 \%$ died, and, of those, $30 \%$ of the deaths were due to bladder cancer. No statistically significant differences were found for these long-term end points.

Conclusions: For prophylaxis of recurrence, maintenance BCG is required to demonstrate superiority to MMC. Prior intravesical chemotherapy was not a confounder. There were no statistically significant differences regarding progression, overall survival, and cancer-specific survival between the two treatments.

(C) 2009 Published by Elsevier B.V. on behalf of European Association of Urology.

\section{Introduction}

Almost a century ago, prospects of the immunotherapeutic treatment of cancer were published by William Coley after analysing case reports on the effect that infectious disease had on existing solid tumours [1]. Coley's toxins, consisting of inactive bacterial cultures, became a wellknown remedy. This medicine did not stand the test of time, but the idea of treating cancer by manipulating the immune system had been born and became a prospering field of research.

Until now, the most frequently used medicine of this kind is bacillus Calmette-Guérin (BCG), with the indication being intravesical therapy of non-muscle-invasive bladder cancer (NMIBC). Before the US Food and Drug Administration (FDA) approved BCG in 1990, chemotherapy in the form of thiotepa, adriamycin, or mitomycin C (MMC) was used.

Presently published clinical guidelines recommend that patients at intermediate or high risk of recurrence and at an intermediate risk of progression should be treated with BCG or MMC in an adjuvant setting after transurethral resection (TUR). This recommendation is based on meta-analyses showing that chemotherapy delays the time to first recurrence after TUR [2]. No influence of chemotherapy on the time to progression to muscle-invasive disease or survival has been found.
Concerning immunotherapy, three meta-analyses confirmed that BCG after TUR is superior to TUR alone or to TUR and chemotherapy in preventing recurrences [3-5]. Two meta-analyses demonstrated that maintenance BCG prevents, or at least delays, the risk of tumour progression [6-7].

Although most evidence has pointed to the superior efficacy of BCG compared to most chemotherapies, it is not so in comparison with MMC. Several meta-analyses have dealt with this comparison. The Cochrane group performed a meta-analysis of seven trials and concluded that tumour recurrence was significantly reduced with BCG only in the subgroup of patients at high risk of tumour recurrence [8]. This finding is in contrast to another metaanalysis that suggested the superiority of BCG over MMC for prevention of tumour recurrence in the combined data, and particularly in the subgroup of patients treated with maintenance BCG, regardless of the actual tumour risk group (intermediate or high risk) [5]. Another metaanalysis indicated that prior chemotherapy treatment in a large number of the randomised trials biased the results in favour of BCG [9]. The authors concluded that the currently perceived superiority of BCG therapy might, therefore, be an artefact of this phenomenon, since most randomised trials included chemotherapy failures in their chemotherapy treatment arms.

Table 1 - Characteristics of the included trials

\begin{tabular}{|c|c|c|c|c|c|}
\hline Reference & BCG strain/dosage & MMC dosage & Treatment duration & Prior chemo allowed & Comments \\
\hline Rintala et al [13] & Pasteur F & $20-40 \mathrm{mg}$ & Both: $6 w+24 m$ & Yes & $\begin{array}{l}\text { BCG intermediate dose; } \\
91 \text { patients }\end{array}$ \\
\hline Witjes et al [14] & RIVM & $30 \mathrm{mg}$ & $\begin{array}{l}\text { MMC: } 4 w+5 m \\
\text { BCG: } 1-2 \times 6 w\end{array}$ & No & 349 patients \\
\hline Witjes et al [15] & Tice and RIVM & $30 \mathrm{mg}$ & $\begin{array}{l}\text { MMC: } 4 w+5 m \\
\text { BCG: } 1-2 \times 6 w\end{array}$ & No & 437 patients \\
\hline Lamm et al [11] & Tice & $20 \mathrm{mg}$ & Both: $6 \mathrm{w}+12 \mathrm{~m}$ & Yes & 445 patients \\
\hline Krege et al [10] & Connaught & $20 \mathrm{mg}$ & $\begin{array}{l}\text { MMC: } 12 \mathrm{bim}+12 \mathrm{~m} \\
\text { BCG: } 6 \mathrm{w}+4 \mathrm{~m}\end{array}$ & Yes & 215 patients \\
\hline Malmström et al [12] & Pasteur D & $40 \mathrm{mg}$ & Both: $6 \mathrm{w}+10 \mathrm{~m}+4 \mathrm{q}$ & Yes & 250 patients \\
\hline Ojea et al [17] & Connaught & $30 \mathrm{mg}$ & Both: $6 \mathrm{w}+$ fortnightly $\times 6$ & Yes & $\begin{array}{l}\text { BCG } 1 / 3 \text { and } 1 / 6 \text { dose; } \\
430 \text { patients }\end{array}$ \\
\hline Friedrich et al [16] & RIVM & $20 \mathrm{mg}$ & $\begin{array}{l}\text { MMC: } 6 \mathrm{w}+\mathrm{m} \text { to } 3 \mathrm{yr} \\
\text { BCG: } 6 \mathrm{w}\end{array}$ & Yes & 495 patients \\
\hline Di Stasi et al [18] & Pasteur & $40 \mathrm{mg}$ & Both: $6 \mathrm{w}+10 \mathrm{~m}$ & No & $\begin{array}{l}\text { Electromotive; MMC } \\
\text { in one arm; BCG } \\
\text { intermediate dose; } \\
\text { CIS only; } 108 \text { patients }\end{array}$ \\
\hline
\end{tabular}


Table 2 - Patient characteristics

\begin{tabular}{|c|c|c|c|}
\hline & \multicolumn{3}{|c|}{ Treatment } \\
\hline & MMC $(n=1383) n(\%)$ & BCG $(n=1437) n(\%)$ & Total $(N=2820) n(\%)$ \\
\hline \multicolumn{4}{|c|}{ Prior intravesical chemotherapy } \\
\hline No & 1117 (93.6) & $1196(92.9)$ & $2313(93.3)$ \\
\hline Yes & $76(6.4)$ & $91(7.1)$ & $167(6.7)$ \\
\hline Unknown & 190 & 150 & 340 \\
\hline \multicolumn{4}{|l|}{ Tumour status } \\
\hline Primary & $828(71.5)$ & $849(70.0)$ & $1677(70.8)$ \\
\hline Recurrent & $330(28.5)$ & $363(30.0)$ & $693(29.2)$ \\
\hline Unknown & 225 & 225 & 450 \\
\hline \multicolumn{4}{|l|}{ Number of tumours } \\
\hline Solitary & $598(53.3)$ & $571(48.9)$ & $1169(51.0)$ \\
\hline Multifocal & $524(46.7)$ & $597(51.1)$ & $1121(49.0)$ \\
\hline Unknown & 261 & 269 & 530 \\
\hline \multicolumn{4}{|l|}{ Stage } \\
\hline Ta & $726(55.3)$ & $708(51.7)$ & $1434(53.5)$ \\
\hline T1 & $538(41.0)$ & $601(43.9)$ & $1139(42.5)$ \\
\hline CIS & $37(2.8)$ & $48(3.5)$ & $85(3.2)$ \\
\hline Dysplasia & $11(0.8)$ & $12(0.8)$ & $23(0.8)$ \\
\hline Unknown & 71 & 68 & 139 \\
\hline \multicolumn{4}{|l|}{ Grade } \\
\hline Grade 0 & $4(0.3)$ & $3(0.2)$ & $7(0.3)$ \\
\hline Grade 1 & $332(25.2)$ & $339(25.0)$ & $671(25.1)$ \\
\hline Grade 2 & $766(58.1)$ & $794(58.5)$ & $1560(58.3)$ \\
\hline Grade 3 & $217(16.5)$ & $221(16.3)$ & $438(16.4)$ \\
\hline Unknown & 64 & 80 & 144 \\
\hline \multicolumn{4}{|l|}{ CIS } \\
\hline No & $1181(87.0)$ & $1255(88.4)$ & $2436(87.7)$ \\
\hline Yes & $177(13.0)$ & $164(11.6)$ & $341(12.3)$ \\
\hline Unknown & 25 & 18 & 43 \\
\hline \multicolumn{4}{|l|}{ Risk group } \\
\hline Low risk & $44(3.3)$ & $48(3.5)$ & $92(3.4)$ \\
\hline Intermediate risk & $964(73.3)$ & 1019 (74.7) & $1983(74.0)$ \\
\hline High risk & 307 (23.3) & $297(21.8)$ & $604(22.5)$ \\
\hline Unknown & 68 & 73 & 141 \\
\hline \multicolumn{4}{|l|}{ BCG maintenance } \\
\hline No & $770(55.7)$ & $726(50.5)$ & $1496(53.0)$ \\
\hline Yes & $613(44.3)$ & $711(49.5)$ & $1324(47.0)$ \\
\hline
\end{tabular}

Table 3 - End points

\begin{tabular}{|c|c|c|c|}
\hline & \multicolumn{3}{|c|}{ Treatment } \\
\hline & MMC $(n=1383) n(\%)$ & BCG $(n=1437) n(\%)$ & Total $(N=2820) n(\%)$ \\
\hline \multicolumn{4}{|c|}{ Recurrence in the bladder } \\
\hline No & $783(56.6)$ & $821(57.1)$ & $1604(56.9)$ \\
\hline Yes & $600(43.4)$ & $616(42.9)$ & $1216(43.1)$ \\
\hline \multicolumn{4}{|c|}{ CIS recurrence } \\
\hline No & $401(84.8)$ & $502(92.6)$ & $903(89.0)$ \\
\hline Yes & $72(15.2)$ & $40(7.4)$ & $112(11.0)$ \\
\hline Unknown & 910 & 895 & 1805 \\
\hline \multicolumn{4}{|c|}{ Progression to muscle-invasive disease ( $\mathrm{T} 2$ or higher) } \\
\hline No & $720(86.7)$ & $936(89.1)$ & $1656(88.1)$ \\
\hline Yes & $110(13.3)$ & $114(10.9)$ & $224(11.9)$ \\
\hline Unknown & 553 & 387 & 940 \\
\hline \multicolumn{4}{|c|}{ Distant metastases } \\
\hline No & $396(93.0)$ & $491(94.6)$ & $887(93.9)$ \\
\hline Yes & $30(7.0)$ & $28(5.4)$ & $58(6.1)$ \\
\hline Unknown & 957 & 918 & 1875 \\
\hline \multicolumn{4}{|c|}{ Survival status } \\
\hline Alive & $596(71.8)$ & $837(79.7)$ & $1433(76.2)$ \\
\hline
\end{tabular}




\begin{tabular}{|c|c|c|c|}
\hline & \multicolumn{3}{|c|}{ Treatment } \\
\hline & MMC $(n=1383) n(\%)$ & BCG $(n=1437) n(\%)$ & Total $(N=2820) n(\%)$ \\
\hline Dead & $234(28.2)$ & $213(20.3)$ & $447(23.8)$ \\
\hline Unknown & 553 & 387 & 940 \\
\hline \multicolumn{4}{|l|}{ Cause of death } \\
\hline Alive & $596(71.8)$ & 837 (79.7) & $1433(76.2)$ \\
\hline Bladder cancer & $77(9.3)$ & $59(5.6)$ & $136(7.2)$ \\
\hline Other & $150(18.1)$ & $146(13.9)$ & $296(15.7)$ \\
\hline Missing & $7(0.8)$ & $8(0.8)$ & $15(0.8)$ \\
\hline Unknown & 553 & 387 & 940 \\
\hline \multicolumn{4}{|c|}{ Death malignant disease } \\
\hline No & $746(89.9)$ & $983(93.6)$ & $1729(92.0)$ \\
\hline Yes & $77(9.3)$ & $59(5.6)$ & $136(7.2)$ \\
\hline Missing & $7(0.8)$ & $8(0.8)$ & $15(0.8)$ \\
\hline Unknown & 553 & 387 & 940 \\
\hline \multicolumn{4}{|c|}{ Systemic chemotherapy } \\
\hline No & $457(92.3)$ & $553(95.7)$ & $1010(94.1)$ \\
\hline Yes & $38(7.7)$ & $25(4.3)$ & $63(5.9)$ \\
\hline Unknown & 888 & 859 & 1747 \\
\hline \multicolumn{4}{|l|}{ Radiotherapy } \\
\hline No & $467(94.5)$ & $542(93.9)$ & 1009 (94.2) \\
\hline Yes & $27(5.5)$ & $35(6.1)$ & $62(5.8)$ \\
\hline Unknown & 889 & 860 & 1749 \\
\hline \multicolumn{4}{|l|}{ Cystectomy } \\
\hline No & $442(85.0)$ & $539(91.4)$ & $981(88.4)$ \\
\hline Yes & $78(15.0)$ & $51(8.6)$ & $129(11.6)$ \\
\hline Unknown & 863 & 847 & 1710 \\
\hline
\end{tabular}

\section{Time to First Recurrence}

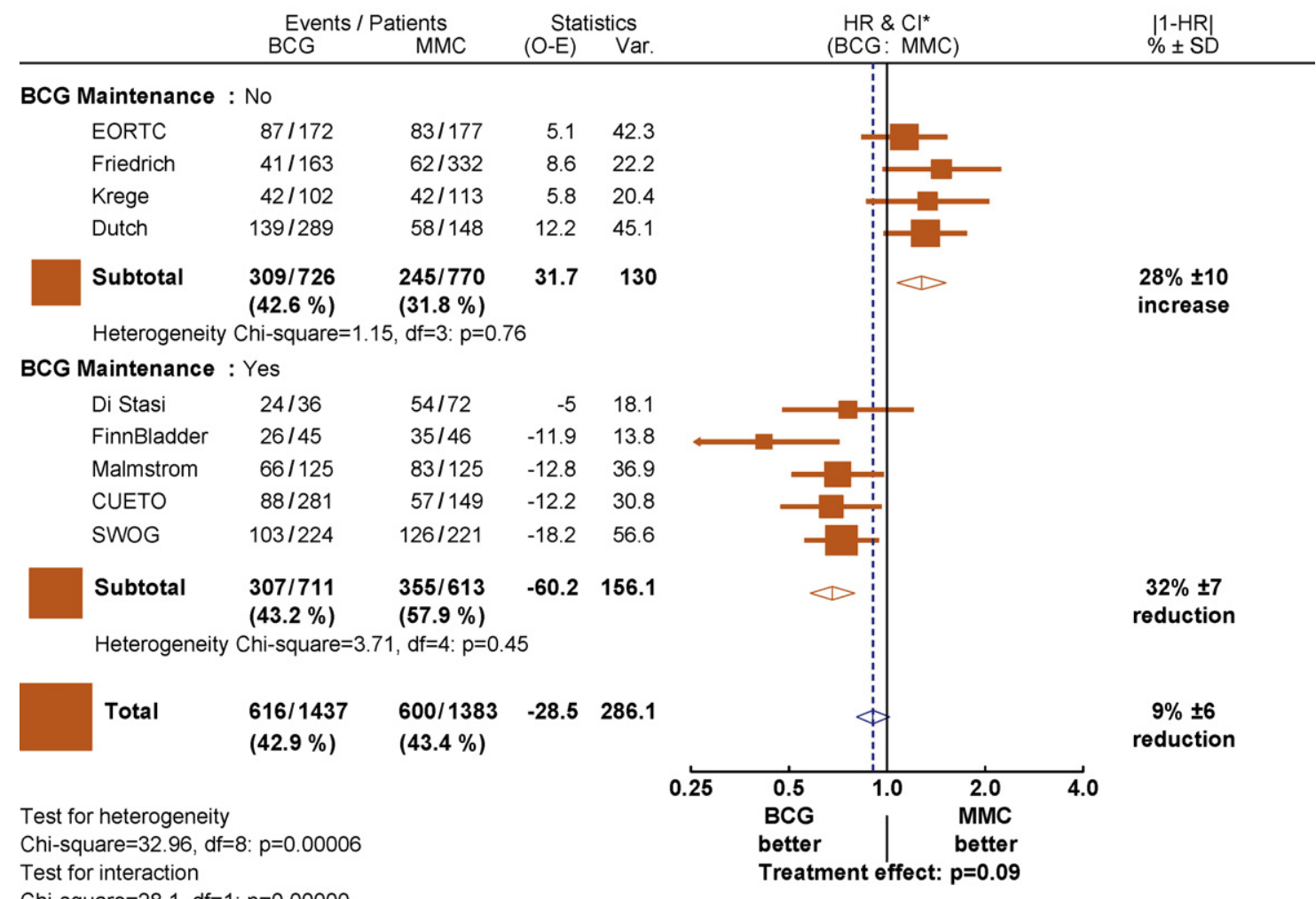

Chi-square $=28.1, \mathrm{df}=1: \mathrm{p}=0.00000$

*95\% Cl everywhere

Fig. 1 - Forest plot of time to first recurrence by study. BCG = bacillus Calmette-Guérin; MMC = mitomycin C. 

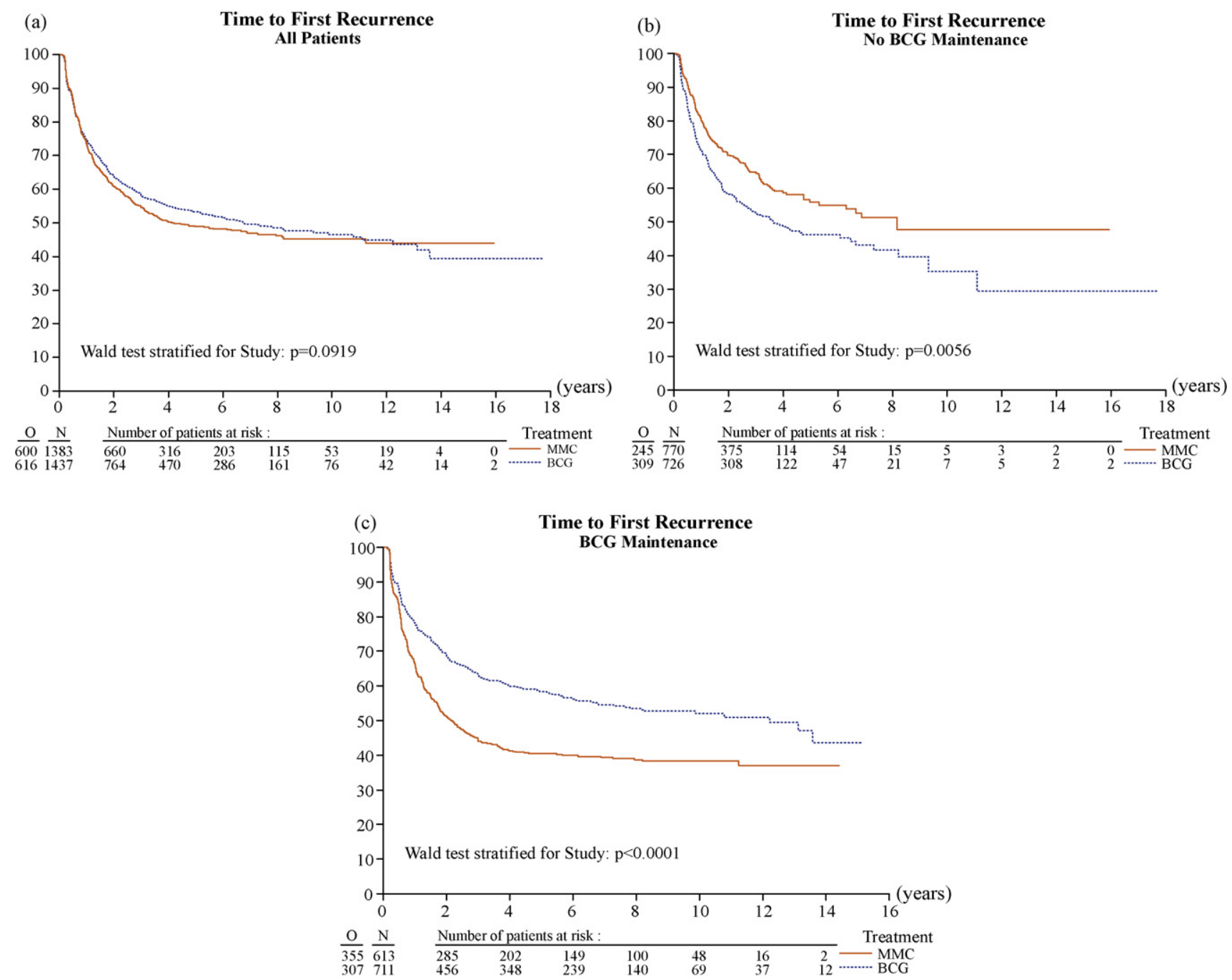

Fig. 2 - Time to first recurrence per treatment group in (a) all patients, (b) no bacillus Calmette-Guérin (BCG) maintenance, and (c) BCG maintenance. MMC = mitomycin C.
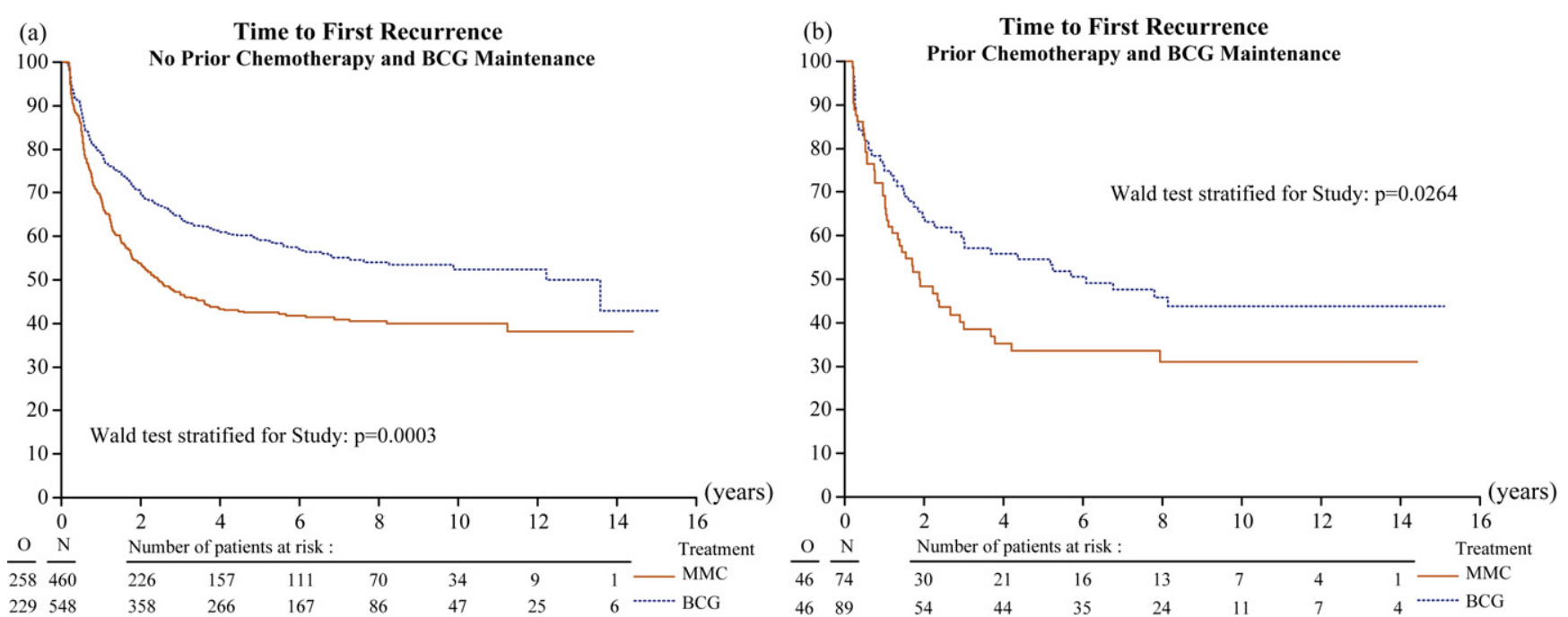

Fig. 3 - Time to first recurrence per treatment group in those with bacillus Calmette-Guérin (BCG) maintenance and (a) no prior chemtherapy or (b) prior chemotherapy. MMC = mitomycin $\mathrm{C}$. 

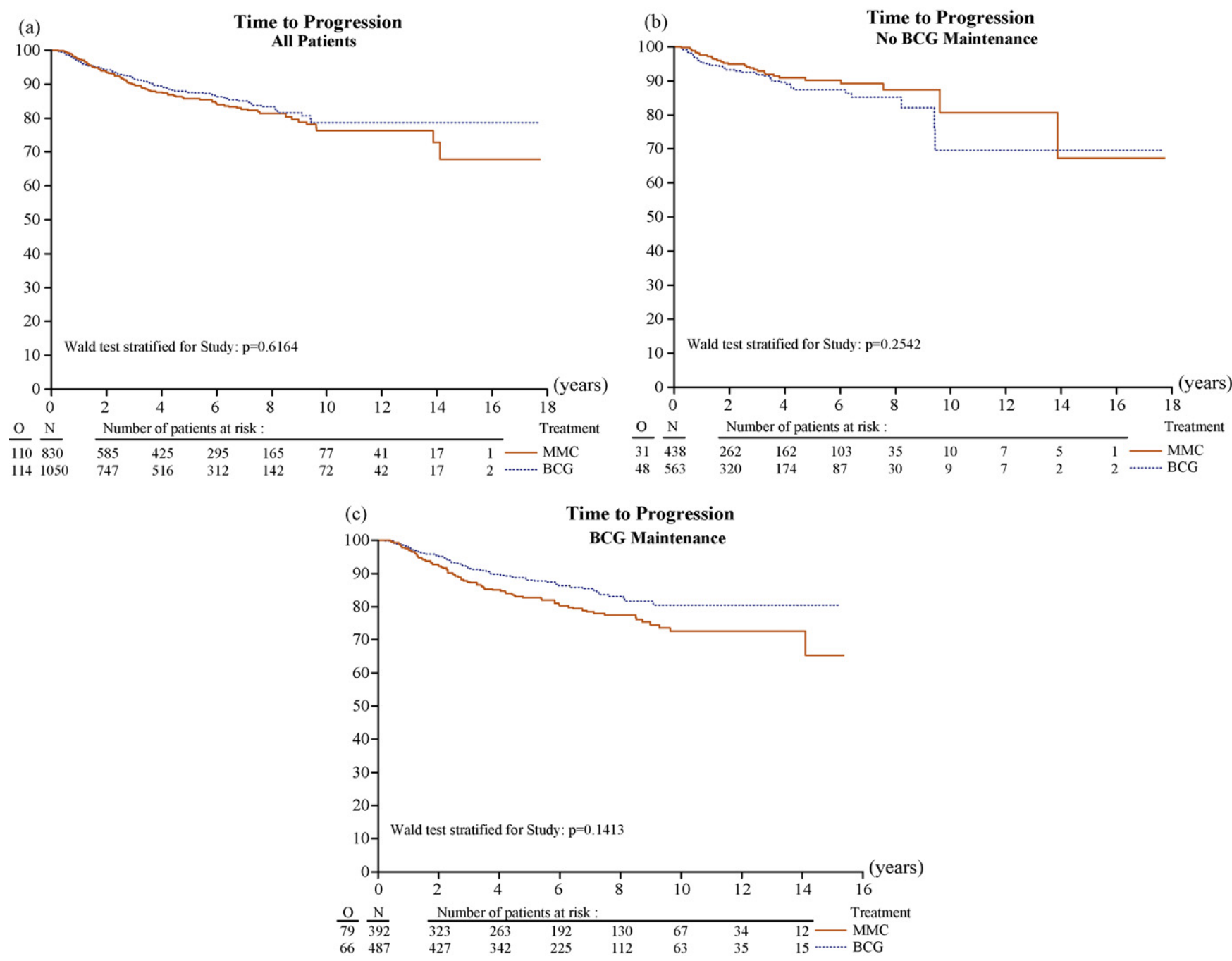

Fig. 4 - Time to progression per treatment group in (a) all patients, (b) no bacillus Calmette-Guérin (BCG) maintenance, and (c) BCG maintenance. MMC = mitomycin $\mathbf{C}$.

Except for Pawinski et al [2], the above-cited metaanalyses have all been quantitative summaries of published data. More reliable information is obtained by a metaanalysis based on the analysis of individual patient data (IPD). The aim of this study was to perform such an IPD meta-analysis to compare the long-term efficacy of intravesical MMC to BCG in patients with NMIBC.

\section{Methods}

\subsection{Inclusion criteria}

Randomised trials comparing TUR plus MMC to TUR plus BCG in patients with NMIBC (stages Ta, T1, Tis) were included in the meta-analysis.

\subsection{Data sources}

We searched the National Library of Medicine (PubMed, at http:// www.ncbi.nlm.nih.gov/sites/entrez) and CancerLit for randomised trials with the above inclusion criteria. These searches were supplemented by hand searching meeting abstracts and proceedings and also by discussion with relevant trial investigators and organisations.

In total, nine trials were identified, and IPD were obtained from all studies [10-18], the main characteristics of which are shown Table 1.

\subsection{Data collection and quality assessments}

The principal investigator responsible for each trial was contacted, and IPD were requested. This request included data on prior intravesical chemotherapy, tumour status (primary or recurrent), number of tumours, stage, concomitant carcinoma in situ (CIS), grade (1973 World Health Organisation [WHO] criteria), date of randomisation, and treatment allocation. Furthermore, the data included end point event status (recurrence, progression to muscle-invasive disease, survival status, and cause of death) and their respective dates, including date of the last follow-up. All data were thoroughly checked for consistency and compared with the data in the most recent publication. Any queries were resolved after discussions with the responsible trial investigator. Reliable data on progression 

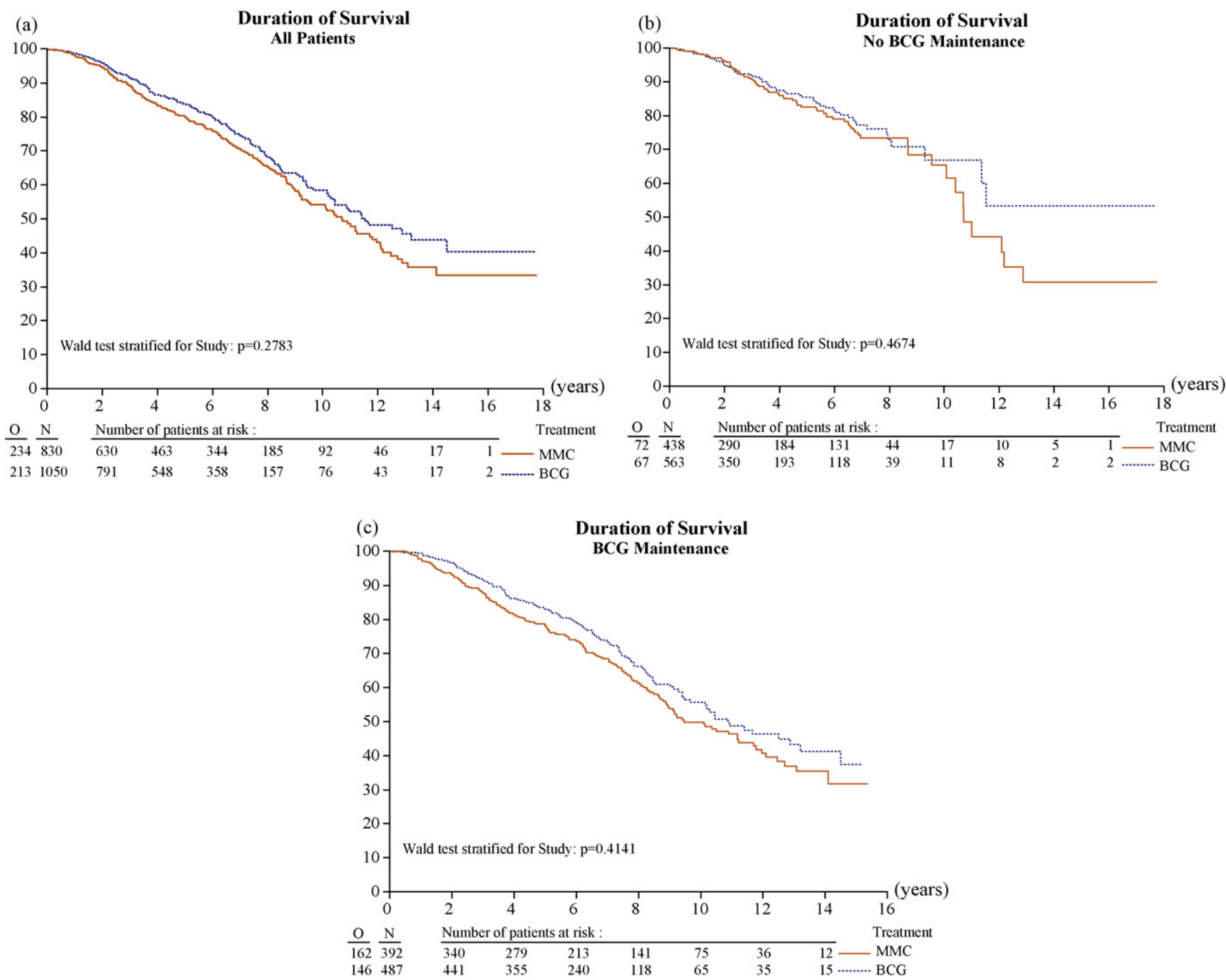

Fig. 5 - Overall duration of survival per treatment group in (a) all patients, (b) no bacillus Calmette-Guérin (BCG) maintenance, and (c) BCG maintenance. MMC = mitomycin C.

and survival or cause of death were not available for two trials $[11,16]$, which have been excluded from the analysis of these end points.

Data that had been updated since publication were received for five studies $[10,11,13,14,18]$.

\subsection{Study outcomes}

The primary end point was time to first recurrence (any stage or grade) in the bladder. Secondary end points were time to progression to muscleinvasive disease (ie, an increase in stage from either Ta or T1 to T2 or higher and duration of overall and cancer-specific survival). Later nonintravesical treatment due to recurrence or progression (such as cystectomy, radiation, or systemic chemotherapy) was also recorded.

\subsection{Statistical analysis}

The efficacy of MMC and BCG was compared for each of the primary and secondary end points described above. Standard fixed-effect metaanalytic procedures for the analysis of individual patient time to event data were used. All analyses were stratified by study. The time to event was estimated by either the Kaplan-Meier technique or cumulative incidence functions, which take competing risks (deaths due to other causes) into account. Time to event distributions were compared using a stratified Wald test or the stratified Gray test for competing risk analyses.

Exploratory subgroup analyses were carried out for the following factors: prior intravesical chemotherapy, tumour status (primary, recurrent), stage, grade, concomitant CIS, maintenance BCG or not, and risk group classification (intermediate risk, high risk). A modified European Association of Urology risk grouping was used: low-risk patients included those with single, primary, Ta G1 tumours; highrisk patients included those with G3 tumours or CIS; and intermediate risk patients included those with all other tumours. Tests for interaction of treatment effect with prior chemotherapy and maintenance BCG were also made. All tests were two-sided using a significance level of 0.05 .

Descriptive analyses were also provided for CIS recurrence, extravesical relapse, cause of death, and nonintravesical treatment after recurrence. 


\section{Results}

Nine trials with 2820 patients were included in the analysis. Patient characteristics were $71 \%$ primary, 54\% Ta, 43\% T1, $25 \% \mathrm{G} 1,58 \% \mathrm{G} 2$, and $16 \% \mathrm{G} 3$, and $7 \%$ had prior intravesical chemotherapy. There were 3\% low-risk, 74\% intermediaterisk, and $23 \%$ high-risk patients. The distribution of patient characteristics and end points in the two treatment arms are detailed in Tables 2 and 3.

Based on a median follow-up of $4.4 \mathrm{yr}$ and a maximum of $17.7 \mathrm{yr}, 43 \%$ recurred. Overall, there was no difference in the risk of recurrence $(p=0.09)$ between BCG and MMC. In the trials with $\mathrm{BCG}$ maintenance, a $32 \%$ reduction in the risk of recurrence for BCG compared to MMC was found $(p<0.0001)$, while there was a $28 \%$ increase in the risk of recurrence $(p=0.006)$ for BCG in the trials without BCG maintenance. The test for interaction was statistically significant at $p<0.0001$ (Figs. 1 and 2).

BCG with maintenance was more effective than MMC in both patients previously treated and those not previously treated with chemotherapy (Fig. 3).

In the subset of seven trials with 1880 patients for whom data on progression, survival, and cause of death were available, $12 \%$ progressed to muscle-invasive disease based on a median follow-up of 4.8 yr. Some $24 \%$ died, and, of those, death was due to bladder cancer in $30 \%$. No statistically significant differences between MMC and BCG were found for these long-term end points (Figs. 4-6).

The results were similar within the different risk groups, indicating that BCG with maintenance was superior to MMC for recurrence but that the progression and mortality rates did not differ significantly between the treatment arms.

\section{Discussion}

In our study, we analysed IPD for patients with NMIBC included in nine trials comparing intravesical MMC to BCG. The relatively high risk of this material is evident, as almost half of the patients had stage T1 tumours. We found that for prophylaxis of recurrence, maintenance BCG was needed to be more effective than MMC. Prior intravesical chemotherapy was not a confounder and, thus, did not bias the results in favour of BCG. There were no statistically significant differences regarding progression, overall survival, and cancer-specific survival between the two treatments.

Two advantages of this analysis are evident compared with earlier ones. This analysis is the first comparing BCG to MMC in which IPD have been used. The superiority of this
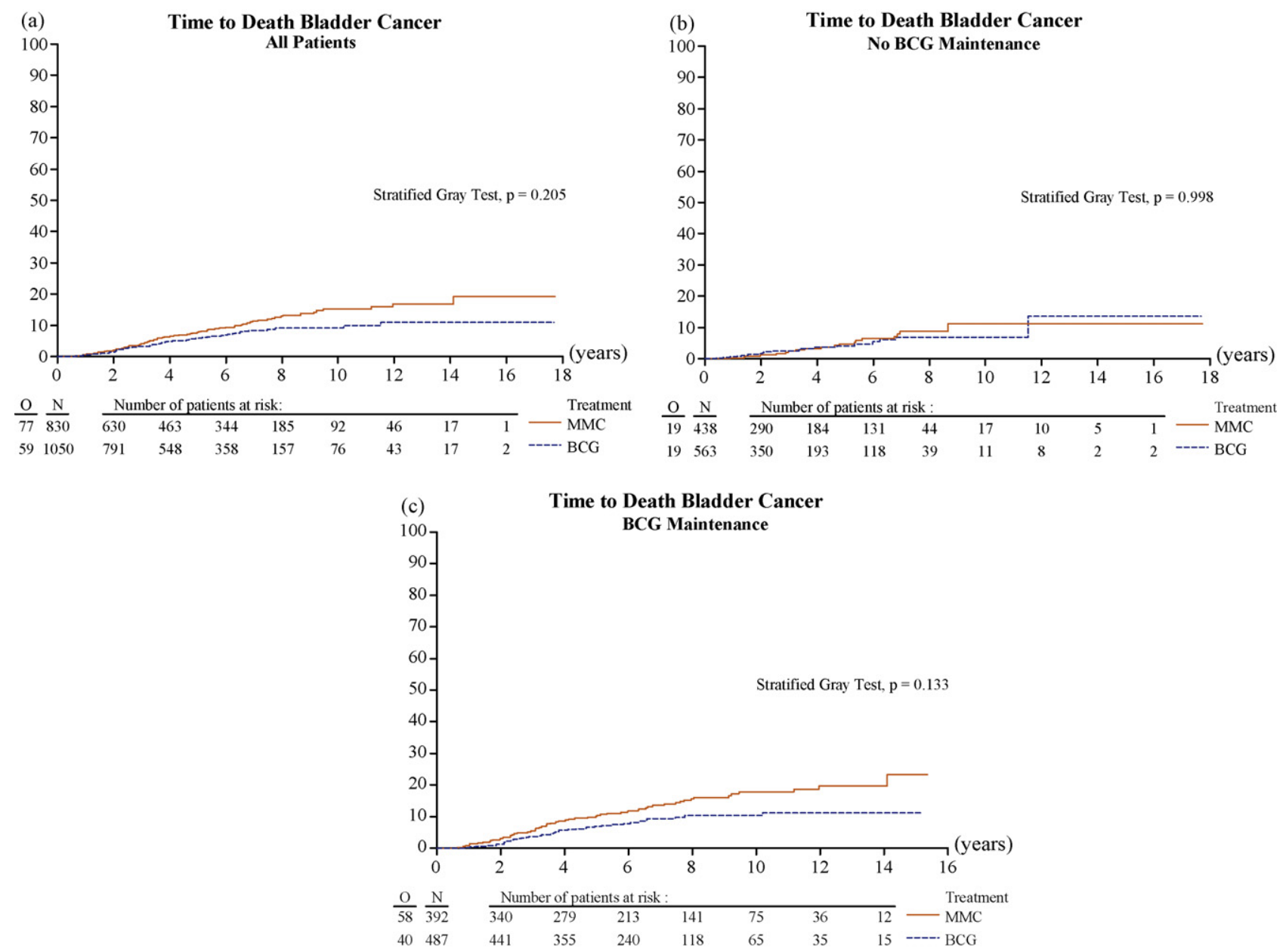

Fig. 6 - Time to death due to bladder cancer per treatment in (a) all patients, (b) no bacillus Calmette-Guérin (BCG) maintenance, and (c) BCG maintenance. MMC = mitomycin $\mathbf{C}$. 
approach as compared to aggregate data from published reports has been emphasised and is the yardstick or gold standard against which other forms of systematic analyses should be measured [19]. Additionally, long-term outcomes have been included. Updated data from five trials were also available for this analysis. The follow-up was longer than that from previous publications, with a median of nearly 5 $\mathrm{yr}$ and a maximum of $>17 \mathrm{yr}$ for progression and death. Some 10-15 yr of follow-up are required to make meaningful comparisons for progression and death due to bladder cancer [20]. In this analysis, only three trials $[12,14,17]$ had such long-term follow-up, indicating that these data are, unfortunately, not usually reported after the initial publication dealing with recurrence.

This study is hampered by the same problems that characterise most meta-analyses of drug trials. This includes patients with differing risks of recurrence and progression, different drug concentrations, different BCG strains, and different treatment and follow-up schedules, as is evident from Table 1. It is also important to stress that long-term follow-up is confounded by the crossover phenomenon, meaning that treatment failures are often treated with the comparison drug at a later date before the long-term end points are reached. The consistency in the results, despite the above issues, indicates that the results are generalisable to the heterogeneous clinical arena.

Despite the large number of patients who have been included, only 224 (12\%) patients progressed and 136 (7\%) died due to bladder cancer, thus limiting the power to detect treatment differences for these end points. Another confounder when analysing progression and survival is that patients can have radical therapy (cystectomy or radiotherapy), despite not progressing; the main reason is that the disease is considered to be resistant to intravesical treatments. Recently, a decrease in survival in patients cystectomised for stage T1 disease has been reported [21]. One possible reason could be the more common use of intravesical therapy nowadays and, thus, a delaying of radical surgery. Therefore, long-term outcomes, as reported in this study, are important to understand the limitations of bladder-sparing therapy.

It is now evident that immunotherapy in the form of BCG has to be given longer than just an induction course. The optimal strain, dosing volume, and duration of maintenance is not known and deserves further study. These factors are even more uncertain concerning chemotherapy, but an early start, within the first postoperative day, is of proven value. The trials included here all [16] had some sort of maintenance MMC, but early postoperative administration was not standard during the time when most of these trials were designed.

Toxicity must always be considered when planning therapy. We did not include data on toxicity in our study for two reasons. First, toxicity is already evident in the short term, and our study focused on long-term events. Second, the toxicity is well known from other trial publications and from an earlier meta-analysis [9]. That meta-analysis showed that approximately $30 \%$ of patients receiving MMC developed local toxicity compared with $44 \%$ with
BCG, and $12 \%$ and 19\% developed systemic side-effects, respectively. Thus, toxicity risk must be weighed against the risks of recurrence and progression to muscle-invasive disease. In the high-risk population, maintenance BCG should be the standard, while in the intermediate-risk group, the less toxic MMC could be considered, with failures being switched to BCG.

\section{Conclusions}

In conclusion, for prophylaxis of recurrence, maintenance BCG is required to demonstrate superiority to MMC. Prior intravesical chemotherapy was not a confounder. There were no statistically significant differences regarding progression, overall survival, and cancer-specific survival between the two treatments.

Author contributions: Per-Uno Malmström had full access to all the data in the study and takes responsibility for the integrity of the data and the accuracy of the data analysis.

Study concept and design: Malmström, Sylvester.

Acquisition of data: Crawford, Krege, Friedrich, Solsona, Rintala, Witjes, Di Stasi.

Analysis and interpretation of data: Malmström, Sylvester.

Drafting of the manuscript: Malmström, Sylvester.

Critical revision of the manuscript for important intellectual content: Malmström, Sylvester.

Statistical analysis: Sylvester.

Obtaining funding: Malmström.

Administrative, technical, or material support: Malmström, Sylvester. Supervision: Malmström, Sylvester.

Other (specify): None.

Financial disclosures: I certify that all conflicts of interest, including specific financial interests and relationships and affiliations relevant to the subject matter or materials discussed in the manuscript (eg, employment/affiliation, grants or funding, consultancies, honoraria, stock ownership or options, expert testimony, royalties, or patents filed, received, or pending), are the following: Dr. Malmström served as a consultant to Kyowa Hakko Kirin UK, Ltd in 2008.

Funding/Support and role of the sponsor: Data collection was supported by Kyowa Hakko Kirin UK, Ltd.

Acknowledgement statement: The authors acknowledge Ulf Hellström at Uppsala Clinical Research Centre, Sara Lammers from Essen, Germany, and Catherine Tangen and Derek Raghavan from Southwest Oncology Group for help with data extraction and queries.

\section{References}

[1] Coley W. The treatment of cancer. Guy's Hosp Gaz 1911;26:7-14.

[2] Pawinski A, Sylvester R, Kurth KH, et al. A combined analysis of European Organization for Research and Treatment of Cancer, and Medical Research Council randomized clinical trials for the prophylactic treatment of stage TaT1 bladder cancer. European Organization for Research and Treatment of Cancer Genitourinary Tract Cancer Cooperative Group and the Medical Research Council Working Party on Superficial Bladder Cancer. J Urol 1996;156:1934-40.

[3] Shelley MD, Kynaston H, Court J, et al. A systematic review of intravesical bacillus Calmette-Guérin plus transurethral resection 
vs transurethral resection alone in Ta and T1 bladder cancer. BJU Int 2001;88:209-16.

[4] Han RF, Pan JG. Can intravesical bacillus Calmette-Guérin reduce recurrence in patients with superficial bladder cancer? A metaanalysis of randomized trials. Urology 2006;67:1216-23.

[5] Bohle A, Jocham D, Bock PR. Intravesical bacillus Calmette-Guerin versus mitomycin $C$ for superficial bladder cancer: a formal metaanalysis of comparative studies on recurrence and toxicity. J Urol 2003;169:90-5.

[6] Sylvester RJ, van der Meijden AP, Lamm DL. Intravesical bacillus Calmette-Guerin reduces the risk of progression in patients with superficial bladder cancer: a meta-analysis of the published results of randomized clinical trials. J Urol 2002;168:1964-70.

[7] Böhle A, Bock PR. Intravesical bacille Calmette-Guérin versus mitomycin $C$ in superficial bladder cancer: formal meta-analysis of comparative studies on tumor progression. Urology 2004;63: 682-6.

[8] Shelley MD, Wilt TJ, Court J, et al. Intravesical bacillus CalmetteGuérin is superior to mitomycin $C$ in reducing tumour recurrence in high-risk superficial bladder cancer: a meta-analysis of randomized trials. BJU Int 2004;93:485-90.

[9] Huncharek M, Kupelnick B. Impact of intravesical chemotherapy versus BCG immunotherapy on recurrence of superficial transitional cell carcinoma of the bladder: metaanalytic reevaluation. Am J Clin Oncol 2003;26:402-7.

[10] Krege S, Giani G, Meyer R, et al. A randomised multicenter trial of adjuvant therapy in superficial bladder cancer: transurethral resection only versus transurethral resection plus mitomycin $C$ versus transurethral restion plus bacillus Calmette-Guerin. J Urol 1996;156:962-6.

[11] Lamm DL, Blumenstein BA, Crawford D, et al. Randomised intergroup comparison of bacillus Calmette-Guerin immunotherapy and mitomycin C chemotherapy prophylaxis in superficial transitional cell carcinoma of the bladder. Urol Oncol 1995;1:119-26.

[12] Malmström P-U, Wijkström H, Lundholm C, et al. 5-year follow-up of a randomised prospective study comparing mitomycin $\mathrm{C}$ and bacillus Calmette-Guerin in patients with superficial bladder carcinoma. J Urol 1999;161:1124-7.
[13] Rintala E, Jauhiainen K, Alfthan O, et al. Intravesical chemotherapy (mitomycin C) versus immunotherapy (bacillus Calmette-Guérin) in superficial bladder cancer. Eur Urol 1991;20:19-25.

[14] Witjes JA, van der Meijden APM, Collette L, et al. Long-term followup of an EORTC randomised prospective trial comparing intravesical bacille Calmette-Guerin and mitomycin C in superficial bladder cancer. Urology 1998;52:403-10.

[15] Witjes WPJ, Witjes A, Oosterhof GON, Debruyne FMJ, Dutch South East Cooperative Urological Group. Update on the Dutch Cooperative Trial: mitomycin $\mathrm{C}$ versus bacillus Calmette-Guerin-Tice versus bacillus Calmette-Guerin RIVM in the treatment of patients with pTa-pT1 papillary carcinoma and carcinoma in situ of the urinary bladder. Semin Urol Oncol 1996;14:10-6.

[16] Friedrich MG, Pichlmeier U, Schwaibold H, Conrad S, Huland H. Long-term intravesical adjuvant chemotherapy further reduces recurrence rate compared with short-term intravesical chemotherapy and short-term therapy with bacillus Calmette-Guérin (BCG) in patients with non-muscle-invasive bladder carcinoma. Eur Urol 2007;52:1123-30.

[17] Ojea A, Nogueira JL, Solsona E, et al. A multicentre, randomised prospective trial comparing three intravesical adjuvant therapies for intermediate-risk superficial bladder cancer: low-dose bacillus Calmette-Guerin $(27 \mathrm{mg})$ versus very low-dose bacillus CalmetteGuerin (13.5 mg) versus mitomycin C. Eur Urol 2007;52:1398-406.

[18] Di Stasi SM, Giannantoni A, Stephen RL, et al. Intravesical electromotive mitomycin $C$ versus passive transport mitomycin $C$ for high risk superficial bladder cancer: a prospective randomised study. J Urol 2003;170:777-82.

[19] Chalmers I. The Cochrane collaboration: preparing, maintaining, and disseminating systematic reviews of the effects of health care. Ann N Y Acad Sci 1993;703:156-63.

[20] Cookson MS, Herr HW, Zhang ZF, Soloway S, Sogani PC, Fair WR. The treated natural history of high risk superficial bladder cancer: 15-year outcome. J Urol 1997;158:62-7.

[21] Lambert EH, Pierorazio PM, Olsson CA, Benson MC, McKiernan JM, Poon S. The increasing use of intravesical therapies for stage T1 bladder cancer coincides with decreasing survival after cystectomy. BJU Int 2007;100:33-6. 